\title{
Chronic endometritis and altered embryo implantation: a unified pathophysiological theory from a literature systematic review
}

\author{
Giovanni Buzzaccarini ${ }^{1}$ (D) Amerigo Vitagliano ${ }^{1}$ - Alessandra Andrisani ${ }^{1} \cdot$ Carla Mariaflavia Santarsiero $^{2}$. \\ Rossana Cicinelli ${ }^{2}$. Claudia Nardelli ${ }^{2} \cdot$ Guido Ambrosini $^{1} \cdot$ Ettore Cicinelli $^{2}$
}

Received: 25 June 2020 / Accepted: 22 September 2020 / Published online: 6 October 2020

(C) The Author(s) 2020

\begin{abstract}
Purpose Chronic endometritis (CE) is a frequent hysteroscopic and histological finding which affects embryo transfer implantation during IVF-ICSI cycles. In particular, CE impairs proper decidualization and, subsequently, implantation. Although this correlation has been clearly clarified, a pathophysiological explanation assembling all the studies performed has not been elucidated yet. For this reason, we have structured a systematic review considering all the original articles that evaluated a pathological element involved in CE and implantation impairment.

Methods The authors searched electronic databases and, after screening, collected 15 original articles. These were fully scanned and used to create a summary pathway.

Results CE is primarily caused by infections, which lead to a specific cytokine and leukocyte pattern in order to prepare the uterus to fight the noxa. In particular, the immunosuppression requested for a proper semi-allogenic embryo transfer implantation is converted into an immunoreaction, which hampers correct embryo implantation. Moreover, endometrial vascularization is affected and both irregular vessel density and luminal thickening and thrombosis reduce what we have first identified as endometrial flow reserve. Finally, incorrect uterine wave propagation could affect embryo contact with decidua.

Conclusion This is the first summary of evidence on CE pathophysiology and its relationship with infertility. Understanding the CE pathophysiology could improve our knowledge in embryo transfer success.
\end{abstract}

Keywords Chronic endometritis $\cdot$ Infertility $\cdot$ Pathophysiology $\cdot$ IVF $\cdot$ Embryo transfer $\cdot$ Recurrent pregnancy failures $\cdot$ Recurrent pregnancy loss

\section{Introduction}

Chronic endometritis (CE) is a persistent inflammatory disorder of the endometrial lining, characterized by superficial endometrial edematous change, high stromal cell density, dissociated maturation between epithelium and stroma, and infiltration of endometrial stromal plasmacytes (ESPCs) [1-5]. The pathogenesis of CE seems to be related to a qualitative and quantitative alteration of endometrial microbioma, with

Giovanni Buzzaccarini

giovanni.buzzaccarini@gmail.com

1 Gynecological Clinic, UOS Medically Assisted Procreation, University of Padova, via Nicolò Giustiniani 3, Padova, Italy

2 Second Unit of Obstetrics and Gynecology, Department of Biomedical Sciences and Human Oncology, University of Bari “A. Moro", Piazza G. Cesare 11, Bari, Italy the abnormal proliferation of different types of microorganisms, mainly gram-negative and intracellular bacteria (i.e., Enterococcus faecalis, Mycoplasma, Ureaplasma, Chlamydia, Escherichia coli, and Streptococcus spp.) [2, 6-9]. As proof of the infectious etiology of CE, several studies have found that specific antibiotic cycles can cure $\mathrm{CE}$ in the majority of patients $[1,7,10]$.

In most cases, women with $\mathrm{CE}$ are asymptomatic or display mild disturbances, such as abnormal uterine bleeding (AUB), dyspareunia, pelvic discomfort, and leukorrhea [11-13]. Moreover, CE cannot be identified by ultrasound examination due to a lack of specific ultrasound markers [8]. For these reasons, CE is often overlooked or diagnosed incidentally during the diagnostic workup of different gynecological disorders including AUB, infertility, or chronic pelvic pain [14].

Fluid hysteroscopy plays a central role in the diagnostic challenge of $\mathrm{CE}$. This technique allows the identification of some endometrial modifications that are specific for CE (i.e., 
focal or diffuse micropolyps, stromal edema, focal hyperemia, strawberry aspect, and endometrial hemorrhagic spots), as recently demonstrated by our group [15].

The current gold standard for CE diagnosis is endometrial biopsy with histological analysis, where the detection of plasma cells within endometrial stroma is the main diagnostic marker [7]. A plasma cell is a type of white blood cell which is derived from B lymphocytes; it is capable of secreting immunoglobulins and is the main cell responsible for humoral immunity. Different studies have shown that traditional staining with hematoxylin and eosin (H\&E) may be not sufficiently accurate for highlighting endometrial plasma cells due to their morphological similarities with fibroblasts. Differently, the immunohistochemical staining for $\mathrm{CD} 138$ was associated with lower intra- and interobserver variability between pathologists in the detection of plasma cells and has now become the reference standard technique for diagnosing CE [16-18].

During the last several decades, $\mathrm{CE}$ has attracted a great deal of attention among scientists and fertility care providers due to its potential association with reproductive issues. In particular, several studies have found that $\mathrm{CE}$ is highly prevalent among women suffering from unexplained infertility (from 40.7 to $55.7 \%$ ), recurrent IVF failures (from 13.95 to $57.55 \%$ ), and repeated early pregnancy loss (from 42.9 to $56 \%$ ). Importantly, adequate therapy of CE can lead to a complete normalization of endometrial histology and to the restoration of the reproductive function in women with $\mathrm{CE}$ [19-24].

In recent years, many authors have investigated the possible mechanisms by which CE may hamper the reproductive health of the endometrium, but the pathophysiological pathway has not been fully assessed yet. In this present study, we aimed to provide the first summary of evidence on the pathophysiological mechanisms involved in CE-related reproductive impairment.

\section{Materials and methods}

\section{Study design}

This is a systematic literature review on the pathophysiological mechanisms involved in CE-related reproductive issues. As it was a review of published data, institutional review board approval was not required.

\section{Search strategy}

Electronic databases (ScienceDirect, MEDLINE, Scopus, Embase, the Cochrane Library, Clinicaltrials.gov, EU Clinical Trials Register, and the World Health Organization International Clinical Trials Registry) were searched for articles indexed from the inception to September 2019. The search was conducted adopting the following keywords: "chronic endometritis AND (infertility OR fertile OR fertility OR miscarriage OR implantation failure $O R$ implantation OR endometrial receptivity OR decidualization OR ART OR IVF OR mechanisms OR causes OR pathway OR pathophysiology)." Furthermore, the reference list of all identified articles and reviews were accurately examined to avoid any missing data.

\section{Inclusion criteria}

All the studies assessing the pathophysiology of infertility in women with $\mathrm{CE}$ were evaluated. Chronic endometritis was defined as a chronic inflammation of endometrium, diagnosed by the histologic presence of one or more plasma cells in the endometrial stroma in the entire section. Articles evaluating other types of endometrial inflammation (such as acute, subacute, or tubercular endometritis) were not included.

No restrictions on the year of publication were applied. The search and the selection criteria were restricted to English language and only studies with full text available were considered suitable for inclusion.

We included only original articles both on humans and animals. However, we did not find any animal study which matched our inclusion criteria. Reviews, systematic reviews and meta-analysis were excluded. The studies evaluated were primarily case-control studies, cohort studies, and retrospective studies.

\section{Study selection and data extraction}

The electronic and reference list searches were performed independently by G.B., R.C., and C.M.S. These investigators then screened titles and abstracts. The results were then compared and any disagreement was resolved by discussion with other reviewers (E.C. and A.V.).

\section{Included studies}

The electronic search identified 419 records. Forty-one fulltext articles were evaluated, of which 26 were excluded. A total of 15 manuscripts were considered eligible for inclusion in the study. Table 1 shows the main characteristics of the studies considered.

Fifteen studies performed hysteroscopy (Carvalho 2013 Cicinelli 2008, Cicinelli 2009, Di Pietro 2013 Di Pietro 2018, Kitaya 2010, Kitaya 2014, Kushnir 2016, Liu 2019, Matteo 2009, Mishra 2008, Moreno 2018, Pinto 2015, Wang 2019, Wu 2017). One study performed vaginal swab (Cicinelli 2008). Nine studies performed biopsy via Novak curettage (Cicinelli 2008, Cicinelli 2009, Di Pietro 2013, Kitaya 2014, Matteo 2009, Mishra 2008, Moreno 2018, Wang 2008, Wu 2017). On the other hand, 2 studies 


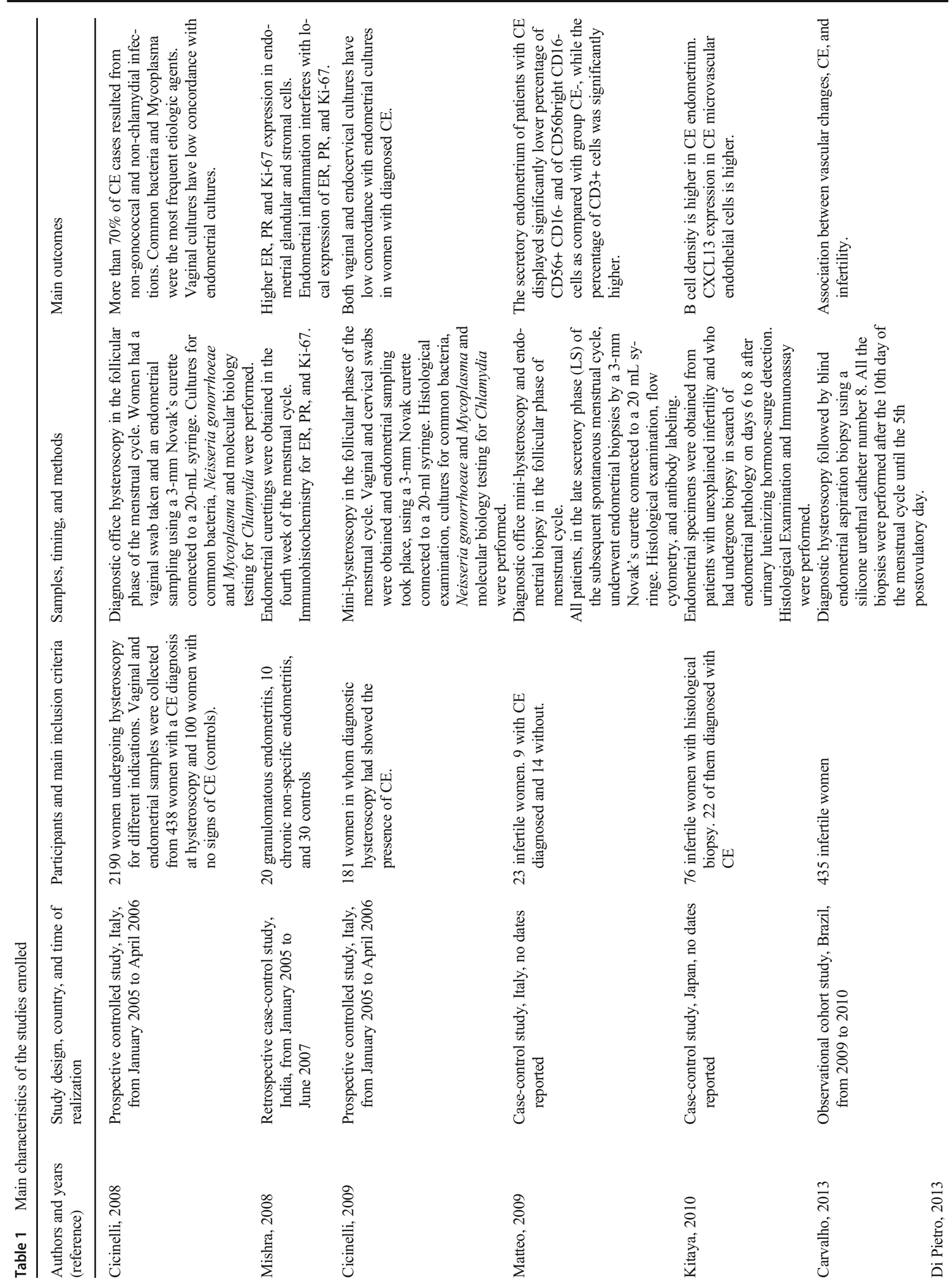




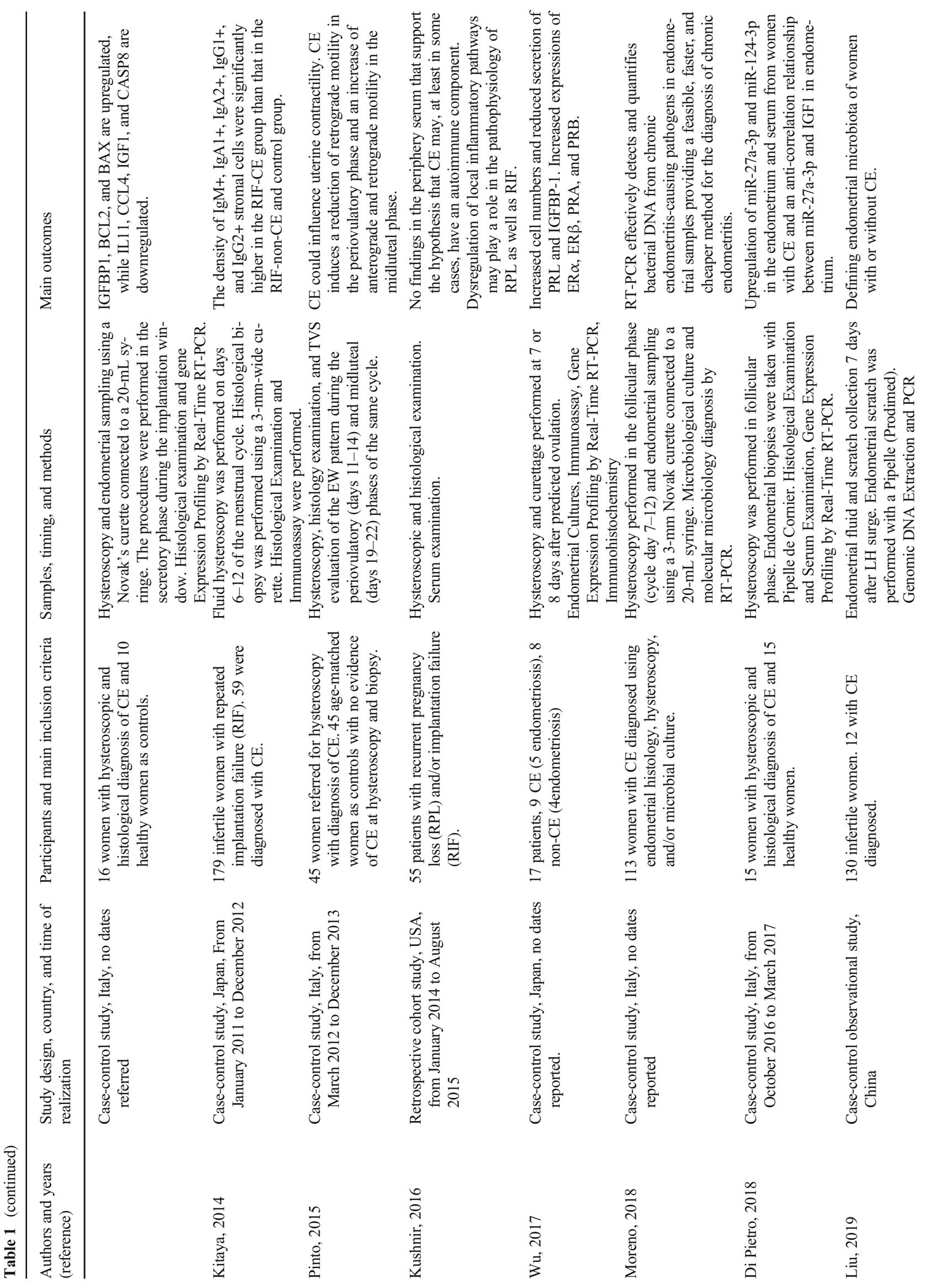




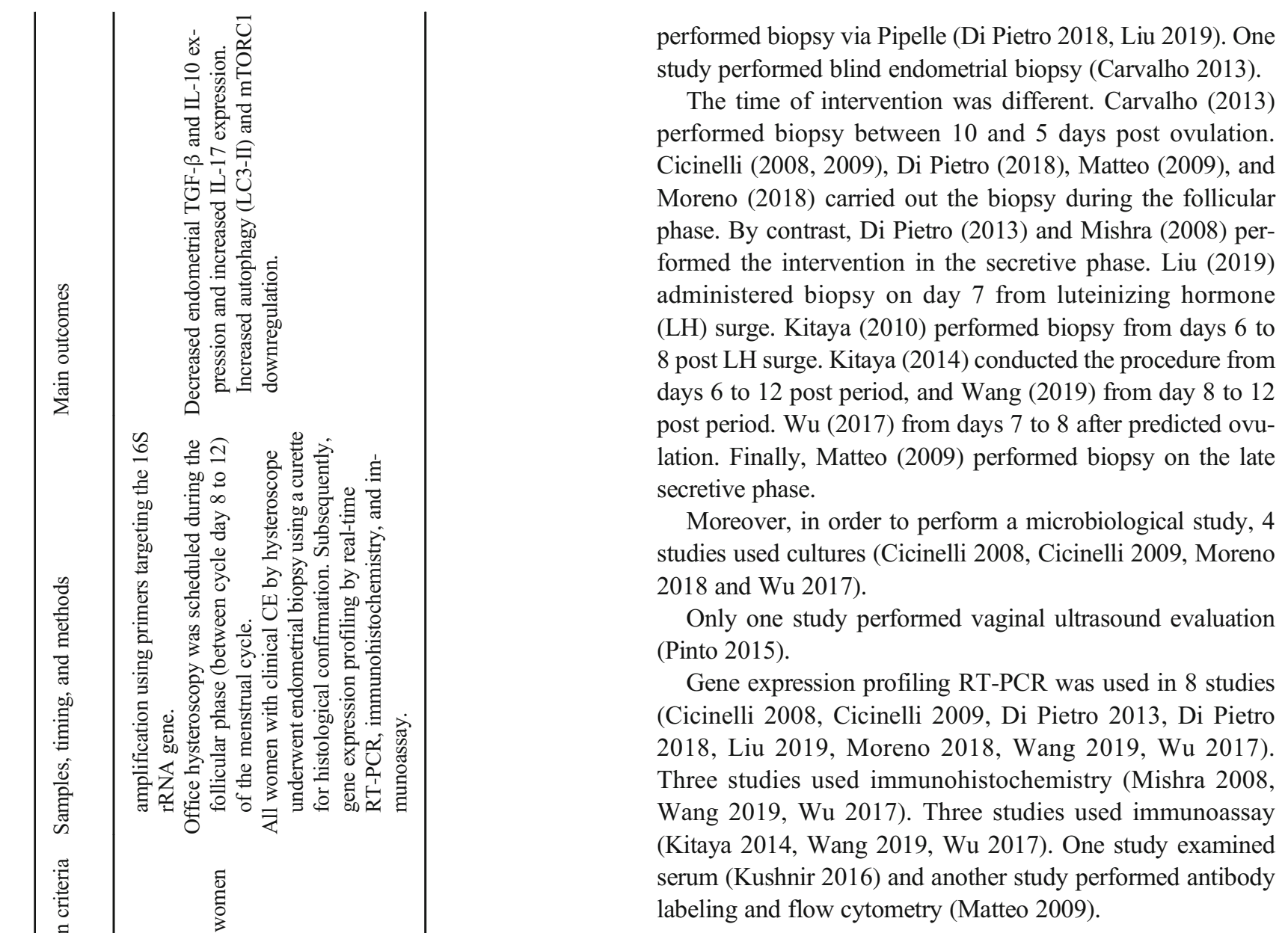

\section{Results and discussion}

Chronic endometritis (CE) is a disease characterized by one main feature: inflammation. Actually, while the biopsy findings are defined and classified, the pathophysiological pathway is still unclear. Moreover, the relationship between $\mathrm{CE}$ and infertility or repeated implantation failure is still under investigation.

Firstly, it must be considered that implantation is the result of a complex interaction between the blastocyst and the endometrium. Different signaling pathways participate in this unique biological link and an appropriate endometrium is requested for the implantation success. This unstable balance can easily be altered by embryonic factors (and this is not our study concern) or endometrial factors. In this case, CE is what inhibits the endometrial ability to achieve a successful implantation.

Our pathophysiological model starts with the following consideration: CE inflammation is mainly caused by infection. Different microorganisms have been detected and various antibiotic protocols have enhanced IVF success after administration in CE infertile women. However, we are of the opinion 
that the infection is only a trigger of a more complex sequence that consists of an academically organized flowchart.

An altered cytokine and chemokine secretion induces altered leukocyte population recruitment. These two conditions impact on uterine contractility, endometrial function in decidualization, and receptivity and vascularization. The main role is played by autophagy, which is necessary for achieving implantation.

\section{Infection and autoimmunity}

In the majority of patients with $\mathrm{CE}$, microbiological analyses (cultures or RT-PCR analyses) are positive for endometrial microorganisms. However, there are also specific cases in which we are unable to identify any microorganisms, or the microorganism is not culturable, raising the doubt of a possible autoimmune pathogenesis of CE. It is to be noted that CE is characterized by plasma cell infiltrates, which are associated with practically all organ autoimmune responses, including rare autoimmune diseases of the reproductive system (i.e., autoimmune oophoritis). To test the hypothesis of CE as an autoimmune condition, a recent study (Kushnir et al. 2016) [25] compared different inflammatory and autoimmune markers between infertile women with CE versus infertile women without $\mathrm{CE}$. The authors failed to demonstrate different values of total immunoglobulins, antinuclear antibodies, thyroid antibodies, and antiphospholipid antibodies between the two groups under comparison $(p>0.05)$, drawing the conclusion that CE does not have a substantial autoimmune component. Although these results need further confirmation, the hypothesis of an autoimmune-driven CE cannot be sustained at present. Accordingly, our model starts considering infections as the main immune trigger for CE.

As is already known, the uterine cavity is not sterile in physiological conditions, but is inhabited by a plethora of microorganisms mainly belonging to Lactobacilli species. Therefore, the isolation of endometrial microorganisms does not necessarily correlate with endometrial inflammation [26]. Cicinelli et al. investigated CE microorganisms by performing both hysteroscopic and histologic exams, in addition to endometrial and vaginal cultures $[2,9]$. The findings can be summarized as follows:

1 CE endometrium showed a prevalence of common bacteria. In particular, streptococci were found in $27.9 \%$ of cases and bacteria from intestinal flora (Enterococcus faecalis and Escherichia coli) were detected in $25.5 \%$ of cases. Ureaplasma urealyticum was detected in $10.0 \%$, and Chlamydia in only $2.7 \%$ of cases. No cases of $N$. gonorrhoeae were found.

2 Endometrial, vaginal, and endocervical cultures in CE women were compared to investigate the percentage of concordance with the etiologic agent. There was a statistically significant difference for Streptococcus, Staphylococcus, E. faecalis, and U. urealyticum (which were found to be more prevalent in vaginal than in endometrial samples).

3 Both vaginal and endocervical cultures have low concordance with endometrial cultures. In particular, positive Staphylococcus endometrial cultures did not have any positive vaginal findings. On the other hand, Chlamydia has a $100 \%$ concordance between endometrial culture and endocervical culture. By contrast, in the majority of cases which proved positive for Ureaplasma and yeast, the microorganisms were also detected at the vaginal level.

However, bacteriological cultures are limited to those microorganisms that are able to grow under traditional culture. About $10 \%$ of cultures were negative in the endometrium suffering from CE (with histological diagnosis), and this was ascribable to the presence of other microorganisms (viruses or non-culturable bacteria) or the presence of autoimmune factors.

Moreno (2018) reported other results. In particular, streptococci were the most abundant bacteria detected (47\%), followed by enterococci (15\%), E. coli (12\%), K. pneumoniae (5\%), staphylococci (3\%), and Mycoplasma hominis (2\%) [8]. These results are quite similar to previous findings and, specifically, it is generally accepted that streptococci are the most prevalent microorganism in CE endometrium. Interestingly, G. vaginalis was detected in $7 \%$ of the samples analyzed and this is in line with Liu 2019, who found an increase in CE endometrium [27]. On the other hand, C. trachomatis and N. gonorrhoeae were undetectable in all tested samples, which is in agreement with the limited role of sexually transmitted disease in the CE pathogenesis of previous studies [8].

Liu (2019) studied CE microbioma on the 7th day after the LH peak (midluteal phase), the most favorable period for possible implantation, with PCR [27]. The main findings are that $\mathrm{CE}$ endometrial cavity shows a lower percentage of Lactobacillus species which are negatively correlated with Gardnerella, Anaerococcus, Finegoldia, and Staphylococcus. These are reported to be associated with preterm delivery and bacterial vaginosis. Lactobacillus species are, indeed, known to inhibit other bacteria by producing hydrogen peroxide and lactic acid. For this reason, Lactobacillus plays a major role as protective bacteria against dangerous microorganisms [28]. Table 2 summarizes the percentage of all the different species detected in these 4 studies [2, 8, 9, 27].

In conclusion, both an increase in dangerous microorganisms and a decrease in protective microorganisms can lead to potential uterine damage. The link between infections and the development of inflammation is a clear pathway step, since a vast number of studies have clarified that organ inflammation diseases can be caused by microorganisms. To clarify, HAV, 
Table 2 microorganism percentage detected at $\mathrm{CE}$ biopsies

\begin{tabular}{|c|c|c|}
\hline Authors and years & Analysis method & Microorganisms detected in $\mathrm{CE}$ endometrium \\
\hline Cicinelli 2008 & $\begin{array}{l}\text { Hysteroscopic and histologic exam, } \\
\text { microorganism cultures. }\end{array}$ & $\begin{array}{l}\text { Streptococci } 27,9 \% \\
\text { Intestinal flora (Enterococcus } \\
\quad \text { faecalis and Escherichia coli) } 2.5 \% \\
\text { Ureaplasma urealyticum } 10 \% \\
\text { Chlamydia } 2.5 \% \\
\text { Staphylococci } \\
\text { Neisseria gonorrhoeae } 0 \%\end{array}$ \\
\hline Cicinelli 2009 & Microorganism cultures, PCR & $\begin{array}{l}\text { Streptococci } 28,7 \% \\
\text { Intestinal flora (Escherichia coli, } \\
\quad \text { Enterococcus faecalis) } 26,6 \% \\
\text { Ureaplasma urealyticum } 11 \% \\
\text { Chlamydia trachomatis } 2,8 \% \\
\text { Staphylococci } 4 \%\end{array}$ \\
\hline Moreno 2018 & PCR & $\begin{array}{l}\text { Streptococci } 47 \% \\
\text { Enterococcus faecalis } 15 \% \\
\text { Escherichia coli } 12 \% \\
\text { Gardnerella vaginalis } 7 \% \\
\text { Staphylococci } 3 \% \\
\text { Mycoplasma hominis } 2 \% \\
\text { Chlamydia } 0 \% \\
\text { Neisseria gonorrhoeae } 0 \%\end{array}$ \\
\hline Liu 2019 & PCR & $\begin{array}{l}\text { (vs non-CE endometrium) } \\
\text { Lactobacilli } \downarrow \\
\text { Atopobium } \uparrow \\
\text { Bifidobacterium } \uparrow \\
\text { Gardnerella } \uparrow \\
\text { Prevotella } \uparrow \\
\text { Stenotrophomonas } \uparrow \\
\text { Others } \uparrow\end{array}$ \\
\hline
\end{tabular}

$\mathrm{HBV}$, and HCV are well-known causes of chronic hepatitis and this can lead both to cirrhosis and liver hepatocarcinoma. On the other hand, Alzheimer's disease is now also related to viral infections such as Human herpesvirus 1 (HHV-1), Cytomegalovirus (CMV), and Human herpesvirus 2 (HHV2) [29]. For this reason, it seems reasonable that an organ which is more prone to extra-corporeal contamination can also develop a chronic inflammatory disease. In particular, the link between infections and inflammation is the first step in our pathway and it is mediated by an interaction between inflammation stimuli such as microbial products, interleukin-1 $\beta$ (IL-1 $\beta$ ), interleukin-6 (IL-6) and tumor necrosis factor- $\alpha$ (TNF- $\alpha)$, and toll-like receptors (TLRs), IL-1 receptor (IL-1R), IL-6 receptor (IL-6R), and the TNF receptor (TNFR) [30]. Lipopolysaccharide, in particular, is the major component of the outer membrane of gram-negative bacteria and the ligand to toll-like receptor 4 expressed on their host cells. For this reason, lipopolysaccharide derived from gram-negative bacteria may be a trigger and mediator of chronic endometritis.

\section{Cytokine dysregulation}

Cytokines are inflammation mediators. Infection causes an aberrant local microenvironment due to an altered secretion of paracrine factors. Moreover, the endometrial microvascular endothelium plays a critical role by mediating the recruitment of leukocytes. In particular, the following signaling pathways have been reported in association with $\mathrm{CE}$ :

1 CE downregulates IL-11 expression. A possible explanation can be found in miR-124-3p upregulation, which is a negative modulator of IL-11 [31, 32]. To clarify, IL-11 activity, binding to IL-11R alpha, helps trophoblast invasion by inducing endometrial epithelial adhesion molecule mRNA expression. Moreover, IL-11 plays a key role in decidualization, since it progresses progesterone-induced decidualization of human endometrial stromal cells, and in endometrial vascularization, promoting angiogenesis and/or remodeling of the maternal vasculature [33]. 
2 CE downregulates CCL-4. CCL-4 is a chemokine that attracts natural killer (NK) cells and macrophages, whose activity is important for implantation, as they produce angiogenic factors, such as vascular endothelial growth factor (VEGF) promoting spiral artery remodeling which supports the implantation of the trophoblast. Specifically, CCL-4 can be responsible for uterine NK recruitment from plasma NK cells [31].

3 CE upregulates IGFBP-1, whose expression is normally increased during decidualization [31]. However, Wu et al. (2017) found a discordant result regarding IGFBP-1 [34], which is downregulated in CE. In addition to this, it must be considered that IGFBP-1 is a modulator of trophoblast invasion, and specifically a negative modulator [35]. Probably, IGFBP-1 is a regulator of decidualization, acting as a damper of this complex interaction between embryo and decidua.

4 CE downregulates IGF-1, which mediates the effects of estrogen on endometrial proliferation during the proliferative phase of the endometrial cycle. An explanation can be found on the role of miR-27a-3p, which is a negative modulator of IGF-1 and is upregulated in CE. A significant anti-correlation ratio was found for miR-27a-3p and IGF-1 [31, 32].

5 CE upregulates both BCL-2 and BAX. BCL-2 inhibits CASP-8, which is a pro-apoptotic gene and has a role in endometrial decidualization. The BCL-2 overexpression in women with $\mathrm{CE}$ makes the endometrial cells more resistant to apoptosis. However, BAX partially counteracts BCL-2 [31]. It is possible that this is related to an unstable balance between proliferation and commitment of endometrial cells (see the "Altered autophagy" section).

6 CE enhances selectin E expression in endometrial microvascular endothelium. Selectin $\mathrm{E}$ is not normally found in microvessels of non-pathological endometrium. In particular, selectin E expression is stimulated by IL1b, TNF-a, and lipopolysaccharide [24]. However, selectin E has a clarified role in trophoblast migration within decidual spiral arterioles. For this reason, we can infer that a correct balance between selectin $\mathrm{E}$ and other selectins ( $\mathrm{L}$ or $\mathrm{P}$ ) is necessary for successful implantation, and an excessive expression of selectin E leads to inappropriate trophoblast invasion [36].

7 CE enhances CXCL13 expression in endometrial microvascular endothelium, when it is normally found only on endometrium surface. CXCL13 expression is only enhanced by lipopolysaccharide. It is likely that CXCL13 plays a role in selective recruitment of circulating naive $\mathrm{B}$ cells into the endometrium and has a key role on sustaining inflammation [24].

8 CE enhances CXCL1 expression in endometrial glandular epithelium, when it is usually not detectable and confined in the stroma. CXCL1 expression is enhanced by lipopolysaccharide [24] and, as above, it can have a key role in attracting stromal B cells into glandular epithelium [37].
9 In CE endometrium, there is an increase in IL-17 expression and a decrease in IL- 10 and TGF- $\beta 1$ expressions than in non-CE endometrium [38]. IL-10 and TGF- $\beta 1$, in particular, are anti-inflammatory modulators and are secreted by Tregs. For this reason, they are both markers and mediators of a decreased inflammatory suppression and uNK cell recruitment [39].

Moreover, there is growing interest regarding the importance of mi-RNA regulation in CE. Recently, mi-RNA composition of exosomes has been studied in cows. In particular, exosomes are intercellular communication medium released by the endometrial epithelium into the uterine cavity and are involved in the transfer of signaling proteins (miRNAs and mRNAs) to either the embryo or adjacent endometrium. A difference between $\mathrm{CE}$ and non-CE endometrium has been identified, which requires further investigation. In particular, 118 miRNAs were found differentially expressed in the exosomes of cows without and those with endometritis. In those with CE, 52 miRNAs were found downregulated and 66 upregulated [40].

Women with CE have an altered endometrial expression in genes involved in inflammatory, cell proliferation, and apoptosis processes. $\mathrm{CE}$ is associated with shifted cytokine milieu toward Th17 over Treg immunity in endometrium. These findings support the notion that $\mathrm{CE}$ is associated with increased pro-inflammatory immune responses, which is often related to poor pregnancy outcomes [38]. CE upregulates both BCL-2 and BAX genes. As apoptosis is an important physiological mechanism in maintaining homeostasis during the menstrual cycle and in early phases of pregnancy, the altered anti- to pro-apoptotic factors ratio in women with $\mathrm{CE}$ could affect pregnancy-mediated tissue remodeling during blastocyst implantation and placental development in the uterus. Moreover, the prevalence of anti-apoptotic effect may explain the abnormal endometrial proliferation that is observed in women with $\mathrm{CE}$, with the formation of endometrial micropolyps and, possibly, macropolyps [31]. Moreover, chronic inflammation may even predispose to endometrial carcinogenesis [41], even if this speculation needs robust scientific confirmation. $\mathrm{CE}$ is associated with the local release of different pro-inflammatory and oncogenic mediators such as nitric oxide (NO), cytokines (IL-1 $\beta$, IL-2, IL-6, and TNF- $\alpha$ ), growth factor, and chemokines. These mediators may make the endometrial inflammatory microenvironment more vulnerable toward tumorigenesis [42].

\section{Leukocyte infiltration}

After cytokine hyper-expression, a new microenvironment is created in the endometrium, whose primary aim is no longer implantation, but immune defense against an exogenous agent. In a normal endometrium, $\mathrm{B}$ cells can be found in 
endometrial stroma, but not in surface epithelium, glandular epithelium, or glandular lumina. In CE, B cells are recruited in the functional layer and single cells can be found between epithelial cells and within gland lumina. On the other hand, T cells, NK cells, macrophages, and neutrophils do not show a different pattern compared with non-pathological endometrium $[24,43,44]$. For this reason, it can be assumed that CE inflammation relies on a $\mathrm{B}$ cell response. More specifically, $\mathrm{CE}$ is characterized by a specific Ig-class expression. CE endometrium has a higher density of Ig-bearing endometrial stromal cells than in physiological endometrium. The density of $\operatorname{IgM}+, \operatorname{IgA} 1+, \operatorname{IgA} 2+, \operatorname{IgG} 1+$, and $\operatorname{IgG} 2+$ is higher, but $\mathrm{IgG} 2+$ is the most predominant and peculiar Ig-class in $\mathrm{CE}$ [45]. In particular, IgG2 is the main effector against bacterial capsular poly-saccharide antigens [46]. For this reason, this unique Ig subclass expression in CE is likely to result from in situ production of endometrial plasmacyte infiltrates as a response to infectious trigger [45].

Over the last 30 years, there has been growing interest in a peculiar class of leukocytes in relation to embryo implantation, namely the uNK. These cells have been detected in areas of stromal decidualization, including progesterone-treated endometrium, intrauterine decidua in ectopic pregnancy, and extrauterine decidua in normal pregnancy. They probably play a role in correct decidualization by promoting adequate vascular remodeling $[47,48]$. In $\mathrm{CE}$, during the endometrial secretory phase, the endometrium showed a lower percentage of uterine NK cell (CD56+ CD16- and CD56 ${ }^{\text {bright }}$ CD16-) [49]. This finding may suggest that a different leukocyte pattern may affect endometrial decidualization in CE and, subsequently, embryo implantation. Furthermore, in order to clarify the immunomodulation role of uNK cells, we considered that uNK cells, but not peripheral blood NK cells, were found to selectively express the genes of immunomodulatory proteins. In particular, they express glycodelin and galectin-1, tetraspanins, integrins, lectin-like receptors, and inhibitory receptors in early pregnancy (KIRs) providing a locally immunosuppressive environment at the maternal-fetal interface [50-53].

Thus, uNK are probably involved in both proper endometrial vascularization and immunomodulation. On the contrary, in $\mathrm{CE}$ endometrium, a decrease in these cells is observed which may probably impair implantation.

With regard to T lymphocytes, Matteo et al. found a higher percentage of $\mathrm{CD} 3+$ cells in $\mathrm{CE}$ endometrium and, in particular, the percentage of CD4 cells resulted significantly higher [49]. Interestingly, previous studies have confirmed the important role of Treg cells in regulating local immunomodulation during the implantation window. The semi-allogenic embryo transplantation is tolerated by a peculiar balance of Th1/Th2 and Th17/Treg cell immune responses [54]. In particular, Tregs suppress maternal alloreactive immune responses against paternal antigens in trophoblast thanks to the production of TGF- $\beta$ and IL-10, antiinflammatory mediators. Moreover, among the CD4+ T cells, approximately 10 to $30 \%$ express the Treg transcription factor FOXP3. A complex interaction between uNK and Tregs and proper vascularization and decidualization are under study. For this reason, an unstable balance of $\mathrm{T}$ cells can affect the acceptation of the embryo by the endometrium [39].

\section{Altered uterine contractility}

Under normal conditions, a varied wave contractility pattern is present in the endometrium for the duration of the menstrual cycle. From an academic perspective, the contractility waves can be divided into three patterns: fundus to cervix (anterograde), cervix to fundus (retrograde), opposing (conflicting waves starting simultaneously on the fundus and the cervix and meeting in the middle of the uterus), not propagating (myometrial activity starting chaotically from different sites), and absent. In addition, cycle-dependent changes can also be observed in the characteristics of endometrial wave (EW) patterns. More specifically, during the early follicular phase anterograde, EWs predominate and are characterized by high amplitude and frequency. For the duration of the periovulatory phase, EWs are, for the most part, retrograde. EW activity is, to a large extent, absent throughout the secretory phase. From a physiologic and clinical perspective, it has been speculated that the purpose of anterograde EW is to empty the uterine cavity during the menstrual and early proliferation phases. It may be that retrograde EW during the periovulatory phase is connected with the active transport of sperm from the vagina to the fallopian tubes, while the quiescent status in the luteal phase may facilitate the process of embryo implantation [55-58].

Pinto et al. in 2015 studied the uterine contractility in nonpregnant women with $\mathrm{CE}$ and demonstrated that cycledependent uterine contractility is altered in women affected by $\mathrm{CE}$ [59]. In particular:

1 CE EW showed a 3.3 times lower occurrence of retrograde contractions during the periovulatory phase compared with non-CE uterus.

2 CE EW in midluteal phase showed a propagating contractile activity that was either anterograde or retrograde in $20 \%$ of cases. By contrast, non-CE EWs were local and non-propagating.

The EWs originate from the junctional zone (JZ), which is the transitional interface between the endometrium and the outer myometrium [60]. Different endometrial and myometrial pathologies can potentially cause alterations of the JZ thickness and activity, such as adenomyosis and fibroids [61]. For this reason, Pinto speculated that even the chronic inflammatory process, which occurs in chronic 
endometritis, could affect JZ functionality. In fact, the altered leukocyte population and the altered pattern of paracrine factors could influence the nearby endometrium of the JZ, inducing an altered contractility which can lead to infertility.

\section{Altered vascularization}

Blood vessels are important players in the inflammatory process. Moreover, they are the principal morphological element of functional polyps, where large caliber arterialized vessels are seen in the functional layer. For this reason, a new interest has grown in detecting relationships between polyps and CE. Starting with this consideration, we considered the following study.

Alterations in blood vessels in CE secretive endometrium were studied by Carvalho (2013) [62]. The chief anatomopathological modifications in CE are, in descending order:

1 High vascular density with endothelial proliferation and swelling associated with hyaline vascular wall thickening with luminal occlusion

2 Hyaline thickening of the vascular wall with luminal occlusion

3 High vascular density with endothelial proliferation and swelling

4 High vascular density with endothelial proliferation and swelling associated with small vessel thrombosis

5 Hyaline vascular wall thickening with luminal occlusion and small vessel thrombosis

6 High vascular density with endothelial proliferation and swelling associated with hyaline vascular wall thickening with luminal occlusion and thrombosis of small vessels

7 High vascular density with endothelial proliferation and swelling associated with hyaline vascular wall thickening with luminal occlusion with luminal occlusion and segmented fibrinoid degeneration

8 Small-vessel thrombosis.

According to the study, in $85.7 \%$ of cases, vascular changes were associated with $\mathrm{CE}$, while $\mathrm{CE}$ without vascular changes was observed in only $7.3 \%$ of cases. It was specifically noted that these vascular alterations are almost identical to the thick-walled vessels along the vascular axis of polyps. As a result of these observations, it can be hypothesized that the vessel axis of polyps may represent a developing stage of vasculopathy related to CE. Furthermore, the close relation between endometritis, polyps, and infertility is currently being studied [63]. The relationship between endometrial polyps and CE has recently been investigated, and one of the principal outcomes has been that EPs are often immunoreactive for CD-138, implying a possible role of chronic endometrial inflammation in their pathogenesis [64]. In all probability, these three diseases share a unique pathological substrate, and inflammation is the most likely candidate. However, further studies are needed to prove this relationship.

These pathological findings have the potential to impede endometrium receptivity, and subsequently implantation, in a range of different ways. Firstly, that increased vessel density is associated with recurrent early pregnancy loss must be taken into consideration. A possible reason for this may be found in the increase of environment oxygenation in the early phase of implantation, owing to the harmful effect of reactive oxygen species. By contrast, normal placental development takes place in a relatively hypoxic environment [48]. Secondly, flow reserve could be impaired by swelling, endothelial proliferation, small-vessel thrombosis, and luminal occlusion. This consideration could be comparable to microcirculation pathology in the myocardium. In this situation, when microvessels are affected by luminal stenosis, coronary flow reserve is reduced [65]. Analogously, it could be conjectured that what we can define as "endometrial flow reserve" can be affected by luminal impairment. Specifically, this blood perfusion could be reduced in instances where it is requested for placental development. To summarize, both excessive and reduced vascular efficiency may lead to a deleterious success for embryo implantation.

\section{Altered decidualization}

Endometrial stromal cells (ESCs) participate in the process of decidualization. Estrogen and progesterone modulate decidua in order to facilitate embryo implantation and IGF-1 and IGF2 are mediators for hormone actions [31]. In particular, estrogen stimulates IGF-I gene expression in the endometrium [66] and IGF-II is upregulated in decidualized endometrium in women treated with levonorgestrel [67]. However, more studies are needed to clarify the relationship between the IGF pathway and sexual hormones in decidualization.

At the beginning of this cascade, it is important to understand that the estrogen receptor (ER) expression reaches a peak in the early proliferative phase with a slight decrease in the late proliferative and early secretory phases in both glandular and stromal endometrium. It then shows a marked decrease in the late secretory phase, specifically in glandular cells. On the other hand, the progesterone receptor (PR) expression increases gradually in the proliferative and secretory phases and decreases in the late secretory phase, especially in the glandular cells [68].

This hormonal pattern is a leading promoter of decidualization. Specifically, decidua allows nutrition and gas and waste exchanges between the fetus and mother in cooperation with the placenta and also produces hormones, growth factors, and cytokines such as prolactin (PRL), corticotrophin-releasing factor (CRF), insulin-like growth factor binding protein-1 (IGFBP-1), and interleukin-15. Decidua 
probably also modulates trophoblast invasion and maternal immune system tolerance [69-71].

Two studies were considered in order to clarify the role of $\mathrm{CE}$ in endometrium decidualization: Wu (2017) and Mishra (2008), who both studied the impact of CE on decidualization $[34,72]$. The main findings can be summarized as follows:

1 PRL and IGFBP-1 are markers of decidualization and are both decreased in CE [34]. Importantly, PRL level expression decrease has been previously correlated with spontaneous early pregnancy loss. In particular, PRL role in embryo implantation can be related to detrimental peptides inhibition (e.g., IL-2) and cytokine stimulation (e.g., TNFa). Consequently, these cytokines can recruit immune cells, sensitize the ESCs to Fas-mediated apoptosis, and promote the release of molecules that interact with the blastocyst and allow a successful implantation [73]. By contrast, IGFBP-1 is part of a more complex pathway, the forkhead transcription factor, forkhead box O1A (FOXO1). In particular, IGFBP-1 plays a role in endometrial cell differentiation [74].

2 In patients suffering from both endometriosis and $\mathrm{CE}$, the concentration of PRL and IGFBP-1 produced tend to be lower compared with that in patients with endometriosis without CE [34].

3 It is likely that the decrease in PRL and IGFBP-1 has its origin in gene expression. The mRNA levels of both, indeed, are lower in CE. As above, in this case, a similar tendency is also noted in patients with endometriosis [34].

4 In ESCs, expressions of ER $\alpha$ and ER $\beta$ (estrogen receptors $\alpha$ and $\beta$ ) and PRA and PRB (progesterone receptors A and $\mathrm{B})$ are higher in $\mathrm{CE}$ endometrium than in non-CE endometrium $[34,72]$.

5 The cell number of decidualized ESCs is increased in CE decidua [34]. In particular, a clear cell proliferation can be noticed if considered Ki-67. Ki-67 is a marker of cell proliferation and its expression in the endometrial cells reaches a peak in the follicular phase and then decreases by half during the early luteal phase and drops during the mid- and late-luteal phase. Ki-67 in the endometrial glandular and stromal cells is significantly higher in both granulomatous and CE than the control group [72].

This data suggests that $\mathrm{CE}$ modifies decidualization. In particular, CE slows down decidua maturation by reducing estrogen and progesterone action. If the real cause is unknown, we can probably observe that the receptors are upregulated and speculate that inflammation may be responsible for sexual-steroid resistance. Previously, it was noticed that the loss of PR activity during early pregnancy resulted in the resorption of implantation sites [75]. Moreover, it has been proven that progesterone promotes ESC differentiation [76]. $\mathrm{ER} \alpha$ and PRA are the predominant isoforms that mediate the critical functions of the uterus for the embryo implantation and maintenance of pregnancy [77, 78]. Taken together, these findings can help hypothesize that estrogen and progesterone action is decreased in $\mathrm{CE}$ endometrium and lead to altered embryo implantation.

To conclude, we can speculate that there are a less differentiation stimulus and a greater potential to proliferation in $\mathrm{CE}$ endometrium, which results in a greater number of ESCs with reduced commitment. As a result, the abnormal expression of sex hormone receptors linked to a greater ESCs number brings about the difficulty for the endometrium to prepare the field for a correct implantation.

\section{Altered autophagy}

Autophagy is a mechanism of lysosome-mediated protein degradation that is necessary in maintaining cellular homeostasis by recycling amino acids, reducing the amount of damaged proteins, and modulating intracellular protein levels in response to extracellular signals. In particular, autophagy has important effects on the induction and modulation of the inflammatory reaction, modulates Treg cells, supports their lineage stability, and increases their life-expectancy [79].

Considering the fact that $\mathrm{CE}$ is associated with increased pro-inflammatory immune responses, it has been demonstrated that in $\mathrm{CE}$ endometrium, the level of microtubuleassociated protein 1A/ 1B-light chain 3 (LC3-II protein) is significantly higher, while the level of mTORC1 is significantly lower than in controls [37].

Firstly, it must be considered that LC3-II is the transmembrane form of LC3, a soluble protein that is distributed ubiquitously in mammalian tissues. LC3 exists in two forms: a cytosolic form (LC3-I) and a lipid phosphatidylethanolamineconjugated form (LC3-II) that is inserted into both inner and outer membranes of the autophagosome. Increased numbers of LC3-containing vesicles and increased LC3 flux indicate active autophagosome formation and clearance [80]. However, LC3 increase can be detrimental for cells and promote cell death which could possibly impair endometrial cells and lead to altered implantation [81].

Conversely, mTOR is a regulator of cellular metabolism and has a crucial role in regulating cellular autophagy [82]. mTOR action relies on integrating signals from the environment to the nucleus for the regulation of cell metabolism, proliferation, survival, and autophagy. Indeed, mTOR action in the placenta is positively correlated with birth weight of the infant [83]. In particular, mTOR is an autophagy negative modulator and modifies inflammatory responses by modulating immunoproteasomal degradation [84]. Furthermore, mTOR has a key role in the post-implantation period and its reduction can lead to reduced feto-placental growth and increased resorption rate [85]. 


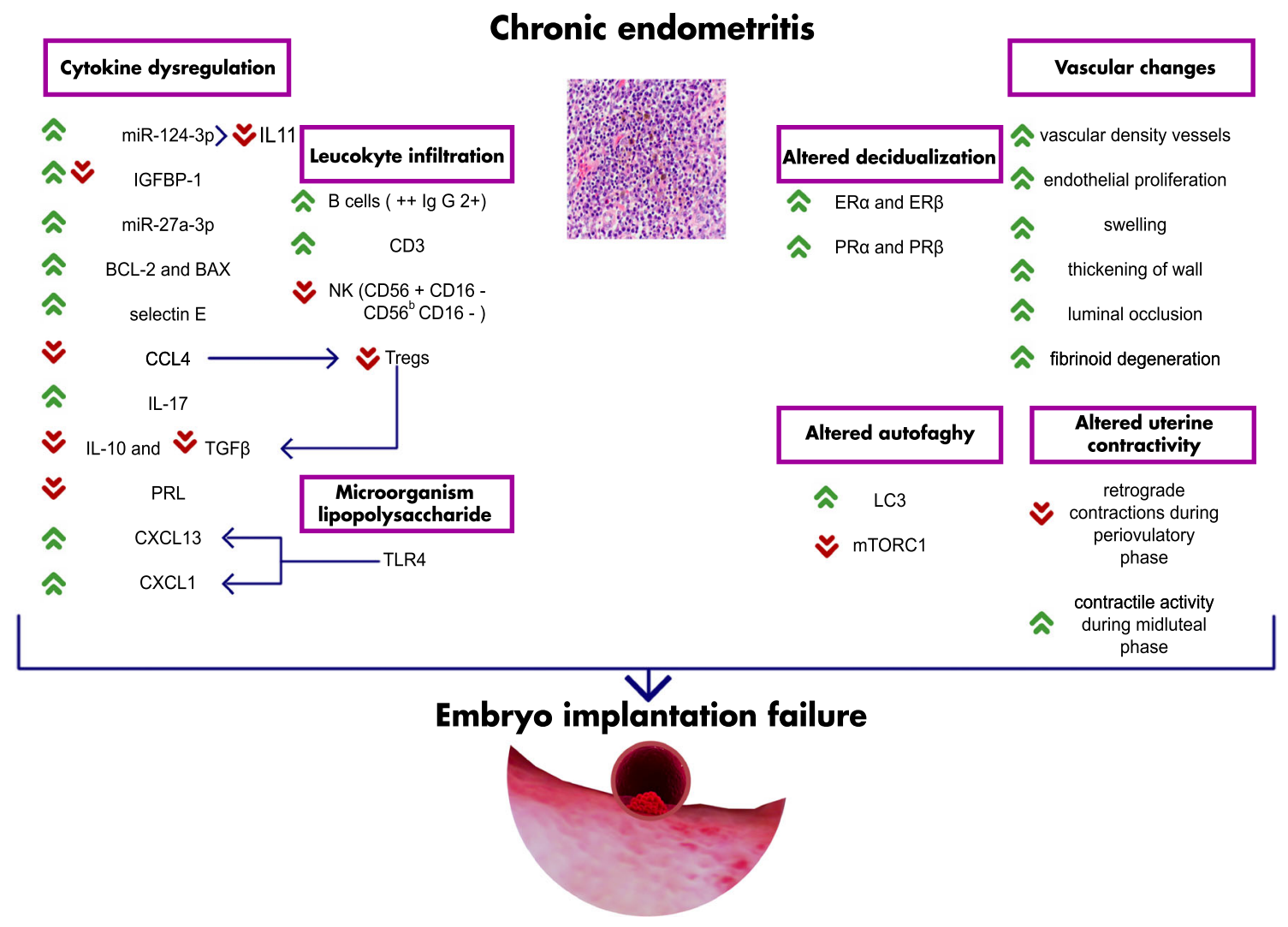

Fig. 1 Chronic endometritis pathway

Our pathophysiological model speculates on the integrating role of both LC3 and mTOR in CE endometrium in implantation. In particular, autophagy is enhanced, and this creates an unstable balance between proteins (or modulators) which should be released, and proteins which should be recycled, leading to an altered environment which is less receptive for implantation. Finally, according to previous findings, autophagy could have an important effect on the induction of inflammatory reaction in $\mathrm{CE}$ and sequential changes in local cytokine milieu.

\section{Strength and limitations}

This review includes all the studies about CE pathophysiology regarding altered implantation. However, most studies were primarily case-control, cohort studies, and retrospective. For this reason, various biases could arise from these study designs.

\section{Conclusion}

To the best of our knowledge, ours is the first summary of evidence on CE pathophysiology and its relationship with altered implantation. CE may affect female fertility in multiple ways, starting from an alteration of endometrial microbiota and continuing with inflammation and its secondary effects.
Abnormal cytokines and leukocyte expression may impair the immune tolerance of the endometrium to the embryo and alter endometrial vascular permeability, potentially damaging embryo viability and trophoblast invasion. Additionally, abnormal uterine contractility during the midluteal phase may inhibit in vivo fertilization and affect transuterine migration of the embryo before implantation. Finally, altered autophagy may affect endometrial cell commitment and impair endometrial decidualization in women with CE. Figure 1 summarizes all the findings and helps visualizing the complexity of our model regarding the chronic endometritis pathway.

Authors' contributions G. Buzzaccarini: Data collection or management, data analysis, manuscript writing, and revision process.

A. Vitagliano: project development, data analysis, and manuscript editing.

A. Andrisani: Manuscript editing.

C.M. Santarsiero: Data collection or management, data analysis, and manuscript writing.

R. Cicinelli: Data collection or management, data analysis, and manuscript writing.

C. Nardelli: Manuscript editing.

G. Ambrosini: Revision process.

E. Cicinelli: Project development, data analysis, and manuscript editing.

Funding Open access funding provided by Università degli Studi di Padova within the CRUI-CARE Agreement. 
Data availability All data are provided with this review.

\section{Compliance with ethical standards}

Conflict of interest The authors declare that they have no conflict of interest.

Open Access This article is licensed under a Creative Commons Attribution 4.0 International License, which permits use, sharing, adaptation, distribution and reproduction in any medium or format, as long as you give appropriate credit to the original author(s) and the source, provide a link to the Creative Commons licence, and indicate if changes were made. The images or other third party material in this article are included in the article's Creative Commons licence, unless indicated otherwise in a credit line to the material. If material is not included in the article's Creative Commons licence and your intended use is not permitted by statutory regulation or exceeds the permitted use, you will need to obtain permission directly from the copyright holder. To view a copy of this licence, visit http://creativecommons.org/licenses/by/4.0/.

\section{References}

1. Cicinelli E, Matteo M, Trojano G, Mitola PC, Tinelli R, Vitagliano A, et al. Chronic endometritis in patients with unexplained infertility: prevalence and effects of antibiotic treatment on spontaneous conception. Am J Reprod Immunol. 2017;79(1).

2. Cicinelli E, De Ziegler D, Nicoletti R. Chronic endometritis : correlation among hysteroscopic, histologic, and bacteriologic findings in a prospective trial with 2190 consecutive office hysteroscopies. Fertil Steril. 2008;89:677-84.

3. Greenwood SMMJ. Chronic endometritis: morphologic and clinical observations. Obstet Gynecol. 1981;58:176-84.

4. Cravello L, Porcu G, D'Ercole C, Roger V, Blanc B. Reconnaître et traiter une endométrite [Identification and treatment of endometritis]. Contracept Fertil Sex. 1997;25(7-8):585-6.

5. Kiviat NB, Wolner-Hanssen P, Eschenbach DA, Wasserheit JN, Paavonen JA, Bell TA, et al. Endometrial histopathology in patients with culture-proved upper genital tract infection and laparoscopically diagnosed acute salpingitis. Am J Surg Pathol. 1990;14(2):167-75.

6. Kamiyama S, Teruya Y, Nohara M, Kanazawa K. Impact of detection of bacterial endotoxin in menstrual effluent on the pregnancy rate in in vitro fertilization and embryo transfer. Fertil Steril. 2004;82:788-92.

7. Liu Y, Phil M, Chen X, Ph D, Huang J, Ph D, et al. Comparison of the prevalence of chronic endometritis as determined by means of different diagnostic methods in women with and without reproductive failure. Fertil Steril. 2018;109:832-9.

8. Moreno I, Cicinelli E, Bs IG, Bs MG, Bau D, Vilella F, et al. The diagnosis of chronic endometritis in infertile asymptomatic women : a comparative study of. Am J Obstet Gynecol. 2018;218:602.e1602.e16.

9. Cicinelli E, De Ziegler D, Nicoletti R, Tinelli R, Saliani N, Resta L, et al. Poor reliability of vaginal and endocervical cultures for evaluating microbiology of endometrial cavity in women with chronic endometritis. Gynecol Obstet Investig. 2009;68:108-15.

10. Kitaya K, Matsubayashi H, Takaya Y, Nishiyama R, Yamaguchi K, Takeuchi T, et al. Live birth rate following oral antibiotic treatment for chronic endometritis in infertile women with repeated implantation failure. Am J Reprod Immunol. 2017;78(5).

11. Quaas A, Dokras A. Diagnosis and treatment of unexplained infertility. Rev Obstet Gynecol. 2008;1:69-77.
12. Cicinelli E, Matteo M, Tinelli R, Pinto V, Marinaccio M, Indraccolo $\mathrm{U}$, et al. Chronic endometritis due to common bacteria is prevalent in women with recurrent miscarriage as confirmed by improved pregnancy outcome after antibiotic treatment. Reprod Sci. $2014 ; 21(5): 640-7$. https://doi.org/10.1177/ 1933719113508817.

13. Park HJ, Kim YS, Yoon TK, Lee WS. Chronic endometritis and infertility. Clin Exp Reprod Med. 2016;43:185-92.

14. Song D, Feng X, Zhang Q, Xia E, Xiao Y, Xie W, et al. Prevalence and confounders of chronic endometritis in premenopausal women with abnormal bleeding or reproductive failure. Reprod BioMed Online. 2018;36:78-83.

15. Cicinelli E, Vitagliano A, Kumar A, Lasmar RB, Bettocchi S, Haimovich S, et al. Unified diagnostic criteria for chronic endometritis at fluid hysteroscopy: proposal and reliability evaluation through an international randomized-controlled observer study. Fertil Steril. 2019;112:162-173.e2.

16. Bayer-Garner IB, Korourian S. Plasma cells in chronic endometritis are easily identified when stained with syndecan-1. Mod Pathol. 2001;14:877-9.

17. Kasius JC, Broekmans FJM, Sie-Go DMDS, Bourgain C, Eijkemans MJC, Fauser BC, et al. The reliability of the histological diagnosis of endometritis in asymptomatic IVF cases: a multicenter observer study. Hum Reprod. 2012;27:153-8.

18. Kannar V, Lingaiah HK, Sunita V. Evaluation of endometrium for chronic endometritis by using syndecan- 1 in abnormal uterine bleeding. J Lab Physicians. 2012;4:69.

19. Vitagliano A, Saccardi C, Litta P, Noventa M. Chronic endometritis : really so relevant in repeated IVF failure? Am J Reprod Immunol. 2017;78(6).

20. Vitagliano A, Saccardi C, Noventa M, Di Spiezio Sardo A, Saccone G, Cicinelli E, et al. Effects of chronic endometritis therapy on in vitro fertilization outcome in women with repeated implantation failure : a systematic review and meta-analysis. Fertil Steril. 2018;103-112.e1.

21. Johnston-MacAnanny EB, Hartnett J, Engmann LL, Nulsen JC, Sanders MM, Benadiva CA. Chronic endometritis is a frequent finding in women with recurrent implantation failure after in vitro fertilization. Fertil Steril. 2010;93:437-41.

22. Vitagliano A, Noventa M, Gizzo S. Autoimmunity, systemic inflammation, and their correlation with repeated implantation failure and recurrent miscarriage: is chronic endometritis the missing piece of the jigsaw?. Am J Reprod Immunol. 2017;77(1). https://doi.org/ 10.1111/aji.12597.

23. Mcqueen DB, Bernardi LA, Stephenson MD. Chronic endometritis in women with recurrent early pregnancy loss and/or fetal demise. Fertil Steril. 2014;101:1026-30.

24. Kitaya K, Yasuo T. Aberrant expression of selectin E, CXCL1, and CXCL13 in chronic endometritis. Mod Pathol. 2010;23(8):113646. https://doi.org/10.1038/modpathol.2010.98.

25. Kushnir VA, Solouki S, Sarig-Meth T, Vega MG, Albertini DF, Darmon SK, et al. Systemic inflammation and autoimmunity in women with chronic endometritis. Am J Reprod Immunol. 2016;75:672-7.

26. Espinoza J, Erez O, Romero R. Preconceptional antibiotic treatment to prevent preterm birth in women with a previous preterm delivery. Am J Obstet Gynecol. 2006;194:630-7.

27. Liu Y, Ko EYL, Wong KKW, Chen X, Cheung WC, Law TSM, et al. Endometrial microbiota in infertile women with and without chronic endometritis as diagnosed using a quantitative and reference range-based method. Fertil Steril. 2019;112:707-717.e1.

28. Santos CMA, Pires MCV, Leão TL, Hernández ZP, Rodriguez ML, Martins AKS, et al. Selection of Lactobacillus strains as potential probiotics for vaginitis treatment. Microbiol (United Kingdom). 2016;162:1195-207. 
29. Sochocka M, Zwolińska K, Leszek J. The infectious etiology of Alzheimer's disease. Curr Neuropharmacol. 2017;15:996-1009.

30. Chen L, Deng H, Cui H, Fang J, Zuo Z, Deng J, et al. Inflammatory responses and inflammation-associated diseases in organs. Oncotarget. 2018;9:7204-18.

31. Di Pietro C, Cicinelli E, Guglielmino MR, Ragusa M, Farina M, Palumbo MA, et al. Altered transcriptional regulation of cytokines, growth factors, and apoptotic proteins in the endometrium of infertile women with chronic endometritis. Am J Reprod Immunol. 2013;69:509-17.

32. Di Pietro C, Caruso S, Battaglia R, Iraci M, La A, Fabrizio F, et al. MiR-27a-3p and miR-124-3p, upregulated in endometrium and serum from women affected by chronic endometritis, are new potential molecular markers of endometrial receptivity. Am J Reprod Immunol. 2018;80:e12858.

33. Dimitriadis E, Menkhorst E, Salamonsen LA, Paiva P. Review: LIF and IL11 in trophoblast-endometrial interactions during the establishment of pregnancy. Placenta. 2010;31:S99-104.

34. Wu D, Kimura F, Zheng L, Ishida M, Niwa Y, Hirata K, et al. Chronic endometritis modifies decidualization in human endometrial stromal cells. Reprod Biol Endocrinol. 2017;15(1):16.

35. Giudice LC. Multifaceted roles for IGFBP-1 in human endometrium during implantation and pregnancy. Ann N Y Acad Sci. 1997;828:146-56.

36. Feng Y, Ma X, Deng L, Yao B, Xiong Y, Wu Y, et al. Role of selectins and their ligands in human implantation stage. Glycobiology. 2017;27:385-91.

37. Wang D, Sai J, Richmond A. Cell surface heparan sulfate participates in CXCL1-induced signaling. Biochemistry. 2003;42:10717.

38. Wang WJ, Zhang H, Chen ZQ, Zhang W, Liu XM, Fang JY, et al. Endometrial TGF- $\beta$, IL-10, IL-17 and autophagy are dysregulated in women with recurrent implantation failure with chronic endometritis. Reprod Biol Endocrinol. 2019;17(1):2.

39. Robertson SA, Care AS, Moldenhauer LM. Regulatory T cells in embryo implantation and the immune response to pregnancy. J Clin Invest. 2018;128:4224-35.

40. Wang X, Tian F, Chen C, Feng Y, Sheng X, Guo Y, et al. Exosome-derived uterine microRNAs isolated from cows with endometritis impede blastocyst development. Reprod Biol. 2019;19: 204-9.

41. Crusz SM, Balkwill FR. Inflammation and cancer: advances and new agents. Nat Rev Clin Oncol. 2015;12:584-96.

42. Qu X, Tang Y, Hua S. Immunological approaches towards cancer and inflammation: a cross talk. Front Immunol. 2018;9:563.

43. Kitaya K, Tada Y, Taguchi S, Funabiki M, Hayashi T, Nakamura Y. Local mononuclear cell infiltrates in infertile patients with endometrial macropolyps versus micropolyps. Hum Reprod. 2012;27: 3474-80.

44. Disep B, Innes BA, Cochrane HR, Tijani S, Bulmer JN. Immunohistochemical characterization of endometrial leucocytes in endometritis. Histopathology. 2004;45:625-32.

45. Kitaya K, Tada Y, Hayashi T, Taguchi S, Funabiki M, Nakamura Y. Comprehensive endometrial immunoglobulin subclass analysis in infertile women suffering from repeated implantation failure with or without chronic endometritis. Am J Reprod Immunol. 2014;72: 386-91.

46. Vidarsson G, Dekkers G, Rispens T. IgG subclasses and allotypes: from structure to effector functions. Front Immunol. 2014;5:1-17.

47. Bulmer JN, Lash GE. Uterine natural killer cells: time for a reappraisal? F1000Research. 2019;8:999.

48. Chen X, Man GCW, Liu Y, Wu F, Huang J, Li TC, et al. Physiological and pathological angiogenesis in endometrium at the time of embryo implantation. Am J Reprod Immunol. 2017;78:1-7.
49. Matteo M, Cicinelli E, Greco P, Massenzio F, Baldini D, Falagario $\mathrm{T}$, et al. Abnormal pattern of lymphocyte subpopulations in the endometrium of infertile women with chronic endometritis. Am J Reprod Immunol. 2009;61:322-9.

50. Koopman LA, Kopcow HD, Rybalov B, Boyson JE, Orange JS, Schatz F, et al. Human decidual natural killer cells are a unique NK cell subset with Immunomodulatory potential. J Exp Med. 2003;198:1201-12.

51. Croy BA, Ashkar AA, Minhas K, Greenwood JD. Can murine uterine natural killer cells give insights into the pathogenesis of preeclampsia? J Soc Gynecol Investig. 2000;12-20.

52. Loke YW, King A, Burrows TD. Decidua in human implantation. Hum Reprod. 1995;10:14-21.

53. Dosiou C, Giudice LC. Natural killer cells in pregnancy and recurrent pregnancy loss: endocrine and immunologic perspectives. Endocr Rev. 2005;26:44-62.

54. Polese B, Gridelet V, Araklioti E, Martens H, d'Hauterive SP, Geenen V. The endocrine milieu and CD4 T-lymphocyte polarization during pregnancy. Front Endocrinol (Lausanne). 2014;5:1-11.

55. Oki T, Douchi T, Maruta K, Nakamura S, Nagata Y. Changes in endometrial wave-like movements in accordance with the phases of menstrual cycle. 2002;28(3):176-81.

56. Kunz G, Noe M, Herbertz M, Leyendecker G. Uterine peristalsis during the follicular phase of the menstrual cycle: effects of oestrogen, antioestrogen and oxytocin. Hum Reprod Update. 1998;4:647-54.

57. Kunz G, Beil D, Deininger H, Wildt L, Leyendecker G. The dynamics of rapid sperm transport through the female genital tract: evidence from vaginal sonography of uterine peristalsis and hysterosalpingoscintigraphy. Hum Reprod. 1996;11:627-32.

58. Fanchin R, Ayoubi JM, Righini C, Olivennes F, Schonauer LM, Frydman R. Uterine contractility decreases at the time of blastocyst transfer. Hum Reprod. 2001;16:1115-9.

59. Pinto V, Matteo M, Tinelli R, Mitola PC. Altered uterine contractility in women with chronic endometritis. Fertil Steril. Elsevier Inc. 2015;103:1049-52.

60. Naftalin J, Jurkovic D. The endometrial-myometrial junction: a fresh look at a busy crossing. Ultrasound Obstet Gynecol. 2009;34:1-11.

61. Tanos V, Balami S, Lingwood L. Junctional zone endometrium alterations in gynecological and obstetrical disorders and impact on diagnosis, prognosis and treatment. Curr Opin Obstet Gynaecol. 2019;31:418-27.

62. Carvalho FM, Aguiar FN, Tomioka R, de Oliveira RM, Frantz N, Ueno J. Functional endometrial polyps in infertile asymptomatic patients: a possible evolution of vascular changes secondary to endometritis. Eur J Obstet Gynecol Reprod Biol. 2013;170(1): 152-6. https://doi.org/10.1016/j.ejogrb.2013.05.012.

63. Kim MR, Ah Kim Y, Jo MY, Hwang KJ, Ryu HS. High frequency of endometrial polyps in endometriosis. J Am Assoc Gynecol Laparosc. 2003;10:46-8.

64. Cicinelli E, Bettocchi S, de Ziegler D, Loizzi V, Cormio G, Marinaccio M, et al. Chronic endometritis, a common disease hidden behind endometrial polyps in premenopausal women: first evidence from a case-control study. J Minim Invasive Gynecol. Elsevier Inc. 2019;26:1346-50.

65. Kaski JC, Crea F, Gersh BJ, Camici PG. Reappraisal of ischemic heart disease: fundamental role of coronary microvascular dysfunction in the pathogenesis of angina pectoris. Circulation. 2018;138: 1463-80.

66. Rutanen EM. Insulin like growth factors in endometrial function. Gynecol Endocrinol. 1998;12:399-406.

67. Rutanen EM. Insulin-like growth factors and insulin-like growth factor binding proteins in the endometrium. Effect of intrauterine levonorgestrel delivery. Hum Reprod. 2000;15:173-81. 
68. Felix JC, Farahmand S. Endometrial glandular proliferation and estrogen receptor content during the normal menstrual cycle. Contraception. 1997;55:19-22.

69. Petraglia F, Tabanelli S, Galassi MC, Garuti GC, Mancini AC, Genazzani AR, et al. Human decidua and in vitro decidualized endometrial stromal cells at term contain immunoreactive corticotropin-releasing factor (CRF) and CRF messenger ribonucleic acid. J Clin Endocrinol Metab. 1992;74:1427-31.

70. Telgmann R, Gellersen B. Marker genes of decidualization: activation of the decidual prolactin gene. Hum Reprod Update. 1998;4: 472-9.

71. Makrigiannakis A, Margioris AN, Chatzaki E, Zoumakis E, Chrousos GP, Gravanis A. The decidualizing effect of progesterone may involve direct transcriptional activation of corticotrophinreleasing hormone from human endometrial stromal cells. Mol Hum Reprod. 1999;5:789-96.

72. Mishra K, Wadhwa N, Guleria K, Agarwal S. ER , PR and Ki-67 expression status in granulomatous and chronic non-specific endometritis. I Obstet Gynaecol Res. 2008;34:371-8.

73. Garzia E, Clauser R, Persani L, Borgato S, Bulfamante G, Avagliano L, et al. Prolactin and proinflammatory cytokine expression at the fetomaternal interface in first trimester miscarriage. Fertil Steril. 2013;100:108-15.

74. Takano M, Lu Z, Goto T, Fusi L, Higham J, Francis J, et al. Transcriptional cross talk between the forkhead transcription factor forkhead box O1A and the progesterone receptor coordinates cell cycle regulation and differentiation in human endometrial stromal cells. Mol Endocrinol. 2007;21:2334-49.

75. Mestre-Citrinovitz AC, Kleff V, Vallejo G, Winterhager E, Saragüeta P. A suppressive antagonism evidences progesterone and estrogen receptor pathway interaction with concomitant regulation of Hand2, Bmp2 and ERK during early decidualization. PLoS One. 2015;10:1-20.

76. Okada H, Sanezumi M, Nakajima T, Okada S, Yasuda K, Kanzaki H. Rapid down-regulation of CD63 transcription by progesterone in human endometrial stromal cells. Mol Hum Reprod. 1999;5:554-8.
77. Conneely OM, Lydon JP. Progesterone receptors in reproduction: functional impact of the a and B isoforms. Steroids. 2000;65:571-7.

78. Hewitt SC, Korach KS. Oestrogen receptor knockout mice: roles for estrogen receptors alpha and beta in reproductive tissues. Repruduction. 2003;125:143-9.

79. Netea-Maier RT, Plantinga TS, van de Veerdonk FL, Smit JW, Netea MG. Modulation of inflammation by autophagy: consequences for human disease. Autophagy. 2016;12:245-60.

80. Tanida I, Ueno T, Kominami E. LC3 and autophagy. Methods Mol Biol. 2008;445:77-88.

81. Kang W, Ishida E, Yamatoya K, et al. Autophagy-disrupted LC3 abundance leads to death of supporting cells of human oocytes. Biochem Biophys Rep. 2018;15:107-14. Published 2018 Aug 21. https://doi.org/10.1016/j.bbrep.2018.08.002.

82. Kim J, Kundu M, Viollet B, Guan KL. AMPK and mTOR regulate autophagy through direct phosphorylation of Ulk1. Nat Cell Biol. 2011;13(2):132-41. https://doi.org/10.1038/ncb2152.

83. Jansson N, Rosario FJ, Gaccioli F, Lager S, Jones HN, Ross S, et al. Activation of placental mTOR signaling and amino acid transporters in obese women giving birth to large babies. J Clin Endocrinol Metab. 2012;98:105-13.

84. Zhang B, Fang L, Wu HM, Ding PS, Xu K, Liu RY. Mer receptor tyrosine kinase negatively regulates lipoteichoic acid-induced inflammatory response via PI3K/Akt and SOCS3. Mol Immunol. 2016;76:98-107.

85. Roberti SL, Higa R, White V, Powell TL, Jansson T, Jawerbaum A. Critical role of mTOR, PPAR $\gamma$ and PPAR $\delta$ signaling in regulating early pregnancy decidual function, embryo viability and fetoplacental growth. Basic Sci Reprod Med. 2018;24:327-40.

Publisher's note Springer Nature remains neutral with regard to jurisdictional claims in published maps and institutional affiliations. 